\title{
Conventional and Molecular Diagnosis of Theileriosis (Theileria annulata) in Cattle in Sulaimani Province, Northern Iraq
}

\author{
Rebwar Bahir Ahmed, ${ }^{1 *}$ Shakhawan Latif Mahmood, ${ }^{1}$ Nawroz Akram Kakrash, ${ }^{1}$ Ihsan K. Zangana, ${ }^{2}$ \\ Mohammed Omar Baba Sheikh ${ }^{3}$ \\ ${ }^{1}$ College of Veterinary Medicine, University of Sulaimani, Sulaimani, Kurdistan Region, Iraq \\ ${ }^{2}$ College of Agriculture, University of Duhok, Duhok, Kurdistan Region, Iraq \\ ${ }^{3}$ Molecular Diagnostic Laboratory, Sulaimani Veterinary Directorate, Sulaimani, Kurdistan Region, Iraq
}

Received 03 May 2021; revised 04 June 2021;

accepted 10 June 2021; available online 22 June 2021

doi:10.24271/psr.26

\begin{abstract}
Theileria annulata has been identified as a major cause of bovine theileriosis in Iraq. The research was designed to reveal the prevalence of Theileriosis (Theileria annulata) in cattle in Sulaimani province, Kurdistan Region- Iraq from the $10^{\text {th }}$ of March 2021 to the $10^{\text {th }}$ of April 2021. Eighty blood samples were collected. Different techniques of parasitic detection, including Microscopic and PCR amplification were used. The results of microscopic examination and PCR assay revealed that $27.5 \%$ and $31.25 \%$ cattle were infected, respectively. The microscopic examination of the Giemsa-stained blood smears of infected erythrocytes revealed the typical morphological structure of piroplasm. According to the phylogenetic analysis of the partial $18 \mathrm{~S}$ ribosomal RNA gene, Theileria field isolates were shown to be related to other $T$. annulata strains, including those from Turkey, Italy, and Pakistan.This study concludes that $T$. annulata is the causative agent of theileriosis of cattle in Sulaimani province and suggests that PCR technique is excellent tools for epidemiological researches and control programs.
\end{abstract}

(C) 2021 Production by the University of Garmian. This is an open access article under the LICENSE

https://creativecommons.org/licenses/by-nc/4.0/

Keywords: T. annulata, Tropical theileriosis, Blood smear, PCR, Sulaimani province

\section{Introduction}

Theileriosis is a haemoprotozoan disease transmitted via biting of ticks and this disease leads to major economic losses in livestock including cattle ${ }^{[1]}$. Ticks (ixodid ticks) act as vectors in spreading of the disease in hot or moderate climates that inject Theileria spp. into the bovine blood ${ }^{[2]}$.

Cattle are generally susceptible to Theileria ${ }^{[3]}$, which includes mostly pathogenic species example, Theileria annulata that leads to tropical theileriosis. Also Theileria parva is another species that causes east coast fever ${ }^{[4]}$.

Tropical theileriosis poses a huge impact on economic losses in dairy industry throughout the world ${ }^{[5]}$. Recent studies reported cattle infection with T. annulata in the Asian countries, including Iraq ${ }^{[6,7]}$, Iran ${ }^{[8,9]}$, Turkey ${ }^{[10]}$, Saudi Arabia ${ }^{[11]}$, and Egypt ${ }^{[12]}$. In the Kurdistan Region of Iraq, reliable and accurate diagnostic methods have rarely been used for epidemiological study; therefore, limited data is known on the epidemiology of tropical

* Corresponding author

E-mail address: rebwar.ahamed@univsul.edu.iq (Instructor).

Peer-reviewed under the responsibility of the University of Garmian. theileriosis ${ }^{[6]}$. In general, clinical signs were somewhat trusted for Theileria diagnosis and it was confirmed by thin blood film stained by Giemsa looking microscopically for piroplasms in erythrocytes or lymph node smear looking for macroschizonts in lymphocyte. However, clinical signs cannot be trustful for diagnosis in many cases because similarity in symptoms are seen between $T$. annulata and other blood parasitic diseases ${ }^{[13,14]}$. In addition, diagnostic method microscopically for sub-clinical and chronic cases needs expertise for reading of the slides. For instance, in case of parasitemias finding of piroplasms may difficult because of their extremely low ratio in the stained blood smears. Therefore, performing of this conventional method for diagnosis of carrier animals is difficult and it requires more time to identify the form of piroplasms in the RBCs ${ }^{[14]}$. To detect of different Theileria species in infected cattle, molecular technique was developed which have a high degree of sensitivity and specificity ${ }^{[15]}$. The prevalence rate of tropical theileriosis caused by of $T$. annulata needs to be determined in cattle in the various areas of Kurdistan Region, therefore the present research was carried out to assess the spread of $T$. annulata in cattle during spring $\left(10^{\text {th }}\right.$ March $-10^{\text {th }}$ April) using conventional microscopy and PCR technique, in Sulaimani, Kurdistan Region of Iraq. 


\section{Material and Methods}

The study was undertaken in the Sulaimani province, Kurdistan Region, Iraq during spring ( $10^{\text {th }}$ March $-10^{\text {th }}$ April $)$. The climate of Sulaimani is variable according to its seasons; temperature in spring is relatively moderate $\left(20^{\circ}-40^{\circ}\right.$ Celsius $)$. The clinical manifestations of theileriosis were noted on some animals during sampling such as high temperature, weakness, lymphnodes swelling in some cases, increased respiration, and nasal discharge. In addition, tick infestations were seen on some examined animals.

\section{1. Samples Collection}

A total number of 80 (male and female) Arabic, local, Simmental and crossbred cattle older than 6 months reared in open management system were screened for haemoprotozoan parasite from various area of Sulaimani province (Tanjaro, Kalar, Smud, Said Sadiq), Kurdistan Region, Iraq. Approximately $6 \mathrm{ml}$ of blood samples from Jugular vein of cattle randomly were collected, $2 \mathrm{ml}$ of the blood were put in tube containing EDTA. The samples were stored and transfer to laboratory in a special designed cool box. Giemsa's stain was done for the blood samples of EDTA tubes to determine of piroplasms microscopically. Then the blood samples were stored at $-70^{\circ} \mathrm{C}$ for PCR test. Ethical standards were considered during sampling.

\section{2. Microscopic Examination (ME)}

After preparation of peripheral blood smears on glass slides, the slides were dried and fixed with methanol for about 5 minutes, then stained by $10 \%$ Giemsa stain for 30 minutes and examined under Oil immersion lens (100 x magnifications).

\section{3. DNA extraction}

The DNA was extracted used $(200 \mu \mathrm{l})$ of the whole blood samples with DNA extraction Kit (ADD BIO INC, DAEGEON Republic of Korea) according to the manufacturer's instructions. The DNAs were eluted into $100 \mu$ Elution buffer at room temperature where they were frozen at $-20^{\circ} \mathrm{C}$ until PCRs performed.

\section{4. PCR amplification and sequencing}

The PCR reactions were performed using specific primer which was designed by $\left[{ }^{16}\right]$ to amplify a $1098 \mathrm{bp}$ fragment of highly conserved regions of $18 \mathrm{~S}$ ribosomal RNA gene in all Theileria spp. 18SrRNA forward primer (AGTTTCTGACCTA TCAG) and 18SrRNA Reverse primer (TTGCCTTAA ACTTCCTTG) were provided by (Macrogen company. Korea). The $18 \mathrm{~S}$ ribosomal RNA gene was amplified using PCR Premix (2X). This master mix provides an entire system for fast, high yield, and reliable single-tube PCR contains: prime taq polymeras 1unite, tris HCL (PH 9.0), PCR enhancer, (NH4)2so4, $4 \mathrm{mM}$ $\mathrm{Mgcl}$, enzyme stabilizer sediment, Loading dye, and $2.0 \mathrm{mM}$ dNTPs mixture (Genetbio, Korea). The reactions were carried out in a $0.2 \mathrm{~mL}$ PCR tube that met the following requirements: $10 \mu \mathrm{L}$ supreme script PCR premix, 5. $\mathrm{LL}$ DNA, 1. $\mu \mathrm{L}$ forward (10 pmol), $1 . \mu \mathrm{L}$ reverse primers $(10 \mathrm{pmol})$, and three $\mu \mathrm{L}$ ultra-pure water to form up a final volume of $20 . \mu \mathrm{L}$. the traditional PCR machine (Hercuvan, USA) was programmed as followed: initial denaturation at $95{ }^{\circ} \mathrm{C}$ for 5 min followed by 40 cycles of $95{ }^{\circ} \mathrm{C}$ for $30 \mathrm{~s}$; annealing at $55^{\circ} \mathrm{C}$ for $30 \mathrm{~s}$, and extension at $72^{\circ} \mathrm{C}$ for $60 \mathrm{~s}$ and a final extension at $72{ }^{\circ} \mathrm{C}$ for 10 -min. After electrophoresis on $1 \%$ agarose gels stained with prime safe dye (Genetbio, Korea) at 100 volt for 60 minutes, the obtained PCR products were visualized under UV illumination. The PCR product for $T$. annulata was estimated to be 1098 bp. Two positive samples' PCR products were sequenced (Macrogen, Republic of Korea) and sent to the national center for bioinformatics and information (NCBI) GenBank.

\section{5. Accession numbers:}

For this analysis, the partial 18SrRNA gene sequences of two field sequences were deposited under the GenBank accession numbers (MW94053 and MW94054).

\section{6. Bioinformatics and sequences analysis}

MEGA.X program was used to bring the sequences together. The ClustaW Multiple Sequence Alignment tool was used to trim and arrange the ribosomal RNA sequences ${ }^{[17]}$. The Neighbor-Joining approach was used to infer the phylogenetic tree and evolutionary relationships ${ }^{[18]}$. In the bootstrap test (1000 replicates), the percentage of duplicate trees in which the related taxa clustered together ${ }^{[19]}$. The Kimura 2-parameter method ${ }^{[20]}$ was used to calculate the evolutionary distances.

\section{Results}

Out of 80 samples, $22(27.5 \%)$ were found positive for tropical theileriosis by conventional microscopic technique using Giemsa staining method; whereas, 25 (31.25\%) samples were detected positive by PCR assay (Table, 1)

Table 1: The prevalence rates of T. annulata infection in cattle using Giemsa staine and PCR methods.

\begin{tabular}{|c|c|c|c|c|}
\hline Species & \multicolumn{2}{|c|}{$\begin{array}{c}\text { Positive sample by } \\
\text { Giemsa staine }\end{array}$} & \multicolumn{2}{|c|}{$\begin{array}{c}\text { Positive sample by } \\
\text { PCR }\end{array}$} \\
\hline Cattle & 22 & $27.5 \%$ & 25 & $31.25 \%$ \\
\hline
\end{tabular}

\section{1. Morphology of Theileria annulata}

The microscopic examination of the Giemsa-stained blood smears of infected erythrocytes revealed the typical morphological structure inside erythrocytes included common shape (ring, Oval, and parachute forms). They were observed as single, double, triple and tetra-forms like inclusion bodies (Figure $1)$.

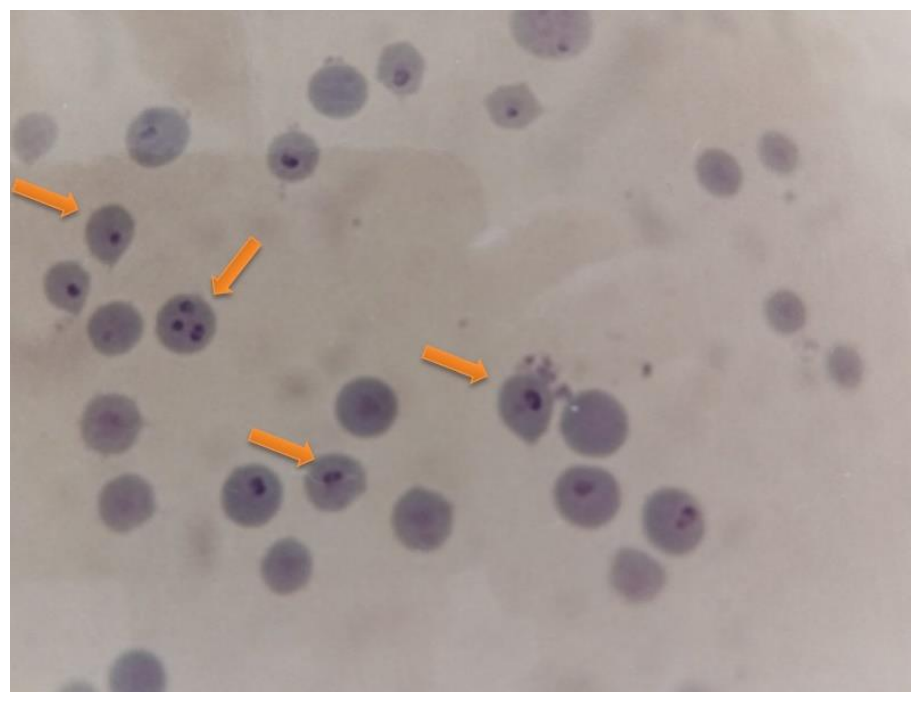

Figure 1: Erythrocytic form of theileria annulata, giemsa stain X 1000 (arrows) 


\section{2. Molecular detection}

PCR is used to identify theileria. PCR analysis showed that 25 of the 80 samples tested were positive, with a band of $1098 \mathrm{bp}$ corresponding to the partial amplification of the $18 \mathrm{~S}$ rRNA gene on agarose gel electrophosis (Figure 2).
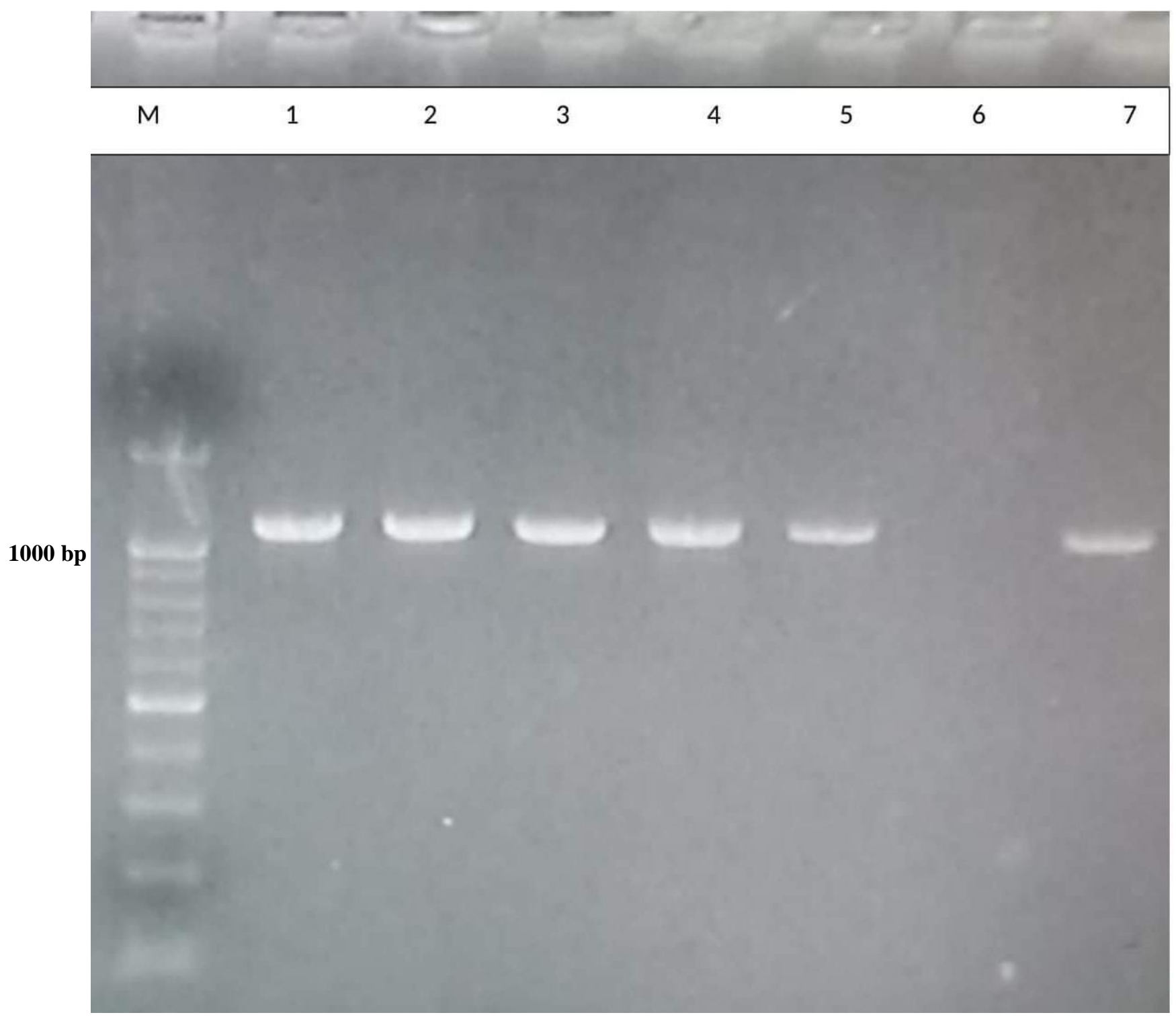

Figure 2: Agarose electrophosis image, which show the PCR product results for Theileria spp of 18S rRNA gene at 1098bp PCR product size,Lane M: Marker 100bp, Lane (1-5+7) are positive samples. Lane 6 Negative sample.

\section{3. Sequencing results}

Two samples of PCR products were sequenced. After sequencing, the strains were given the names (MW940543T.annulata/Sh/3/21, and MW94054-T.annulata/Sh2/3/21). For genetic relatedness, these sequences were linked to $T$. annulata references found in GenBank.

\section{4. Alignment of sequences and phylogenetic analysis}

The two nucleotide sequences of the detected $T$. annulata were $100 \%$ identical and had no diversification. The field sequences isolated with $T$. annulata Turkey and Italy strains with accession numbers (AY508463 and MT341858) exhibited the highest identities of $99.24 \%$ and $99.24 \%$, respectively (Table 2).
When field parasite DNA sequences were compared to other $T$. spp strains in GenBank, $T$. parva was the most closely related, with a similarity of $96.72 \%$, while $T$. sergenti had a similarity of $94.04 \%$. In addition, comparisons of $T$. annulata/Sh/21 sequences with those of an Iraqi strain (Iraq/AN10) indicated no significant differences (Table 2, Figure 3). A partial sequences of the $18 \mathrm{~S}$ rRNA gene from the field strains and representative strains from GenBank were used in phylogenetic analysis to compare and verify the evolutionary ancestry of the field samples. Furthermore, the two field strains were shown to be related to other T. annulata in different countries (Turkey, Italy, and Pakistan). Field isolate sequences are heterologous with T.spp strains like (like T. parva, T. bovis, T.taurotragi, T.capreoli, $T$. sergenti, and T. buffeli ), but only homologous with (T. annulata), according to a phylogenetic tree (Figure 4). 
Table 2: The 18S rRNA gene sequence homology of field T. annulata strains with other Theileria spp. Available in Genbank.

\begin{tabular}{|l|l|l|l|}
\hline Accession no. & country & Theileria spp. & DNA Identities \\
\hline L19082 & South Africa & T.taurotragi & 96.30 \\
\hline AY260172 & Turkey & T.ovis & 94.37 \\
\hline AY726011 & USA & T.capreoli & 94.06 \\
\hline AY661515 & JAPAN & T. sergenti & 94.05 \\
\hline AY661512 & USA/texas & T.buffeli & 94.45 \\
\hline AF013418 & Kenya & T.parva & 96.72 \\
\hline & Iraq/AN10 & T.annulata & 99.61 \\
\hline MT341858 & Italy & T.annulata & 99.24 \\
\hline AY508463 & Turkey & T.annulata & 99.24 \\
\hline
\end{tabular}

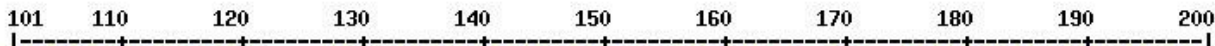

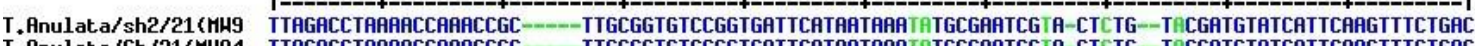

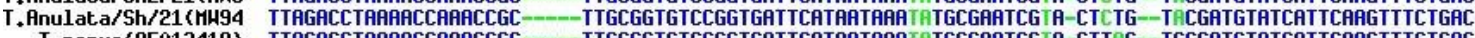

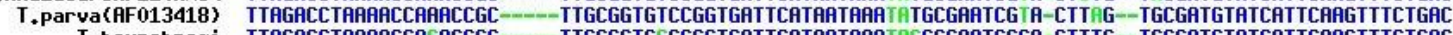

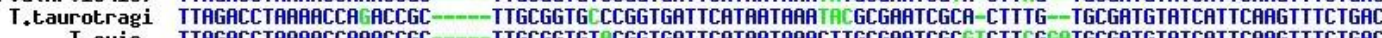

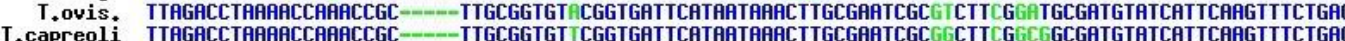
T.capreoli ITAGACCTAAAACCAAACCGC---ITGCGGTGT TCGGTGATTCATAPTAAACTTGCGAATCGCGGCTTCGGCGGCGATGTATCATTCAAGTITCTGAC

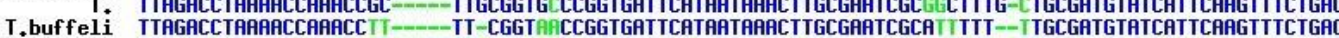

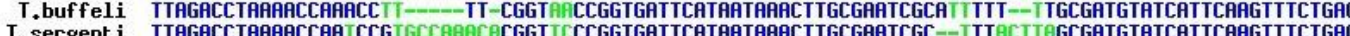

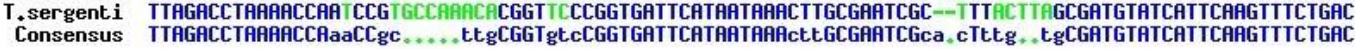

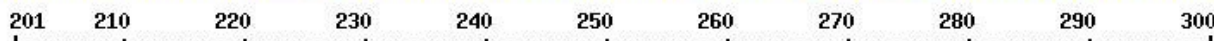

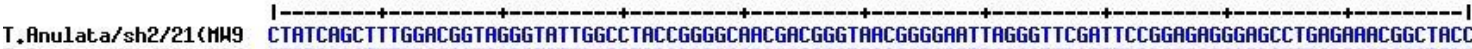
T.Anulata/Sh/21 (rHH94 CTATCAGCTTTGGACGGTAGGGTATTGGCCTACCGGGGCAACGACGGGTAACGGGGAATTAGGGTTCGATTCCGGAGAGGGAGCCTGAGAAACGGCTACC T.parva(AF 013418) CTATCAGCTTTGGACGGTAGGGTATTGGCCTACCGGGGCAACGACGGGTAACGGGGAATTAGGGTTCGATTCCGGAGAGGGAGCCTGAGAAACGGCTACC T.taurotragi CTATCAGCTTTGGACGGTAGGGTATTGGCCTACCGGGGCAGCGACGGGTAACGGGGAATTAGGGTTCGATTCCGGAGAGGGAGCCTGAGAARCGGCTACC T.ovis. CTATCAGCTITGGACGGTAGGGTATTGGCCTACCGGGGCAACGACGGGTAACGGGGAATTAGGGTTCGATTCCGGAGAGGGAGCCTGAGAAACGGCTACC T.capreoli CTATCAGCTITGGACGGTAGGGTATTGGCCTACCGGGGCAACGACGGGTARCGGGGAATTAGGGTTCGATTCCGGAGGGGGAGCCTGAGAPACGGCTACC . CTATCAGCTITGGACGGTAGGGTATTGGCCTACCGGGGCA GCGACGGGTAACGGGGAAT TAGGGTTCGATTCCGGAGAGGGAGCCTGAGAAACGGCTACC

T.buffeli CTATCAGCTITGGACGGTAGGGTATTGGCCTACCGGGGCA GCGACGGGTAACGGGGAATTAGGGTICGATTCCGGAGAGGGAGCCTGAGAAACGGCTACC T. sergenti CTATCAGCTITGGACGGTAGGGTATTGGCCTACCGGGGCAACGACGGGTAACGGGGAATTAGGGTTCGATTCCGGAGAGGGAGCCTGAGAAACGGCTACC Consensus CTATCAGCTITGGACGGTAGGGTATTGGCCTACCGGGGCAaCGACGGGTAACGGGGAPTTAGGGTTCGATTCCGGAGAGGGAGCCTGAGAARCGGCTACC

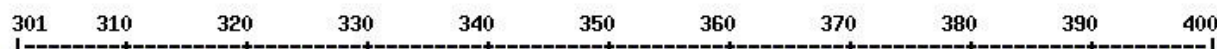

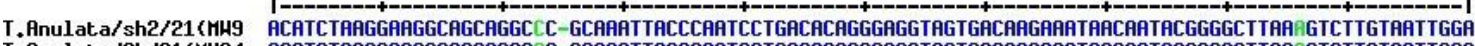

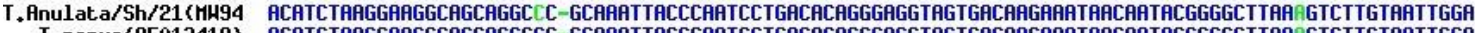

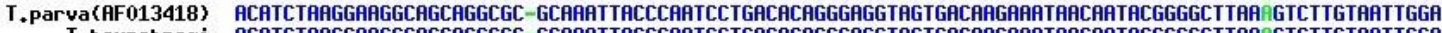

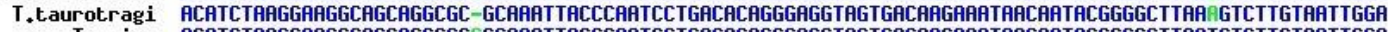

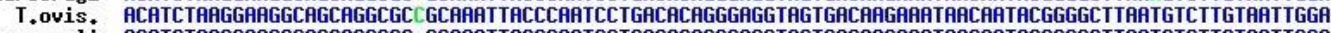

T.capreoli ACATCTAAGGAAGGCAGCAGGCGC-GCAAPTTACCCAATCCTGACACAGGGAGGTAGTGACAAGAAPTAACAATACGGGGCTTPATGTCTTGTAATTGGA T. ACATCTAAGGAAGGCAGCAGGCGC-GCAFATTACCCAATCCTGACACAGGGAGGTAGTGACAAGAAATAACAATACGGGGCTTAATGTCTTGTAATTGGA T,buffeli ACATCTAAGGAAGGCAGCAGGCGC-GCAAPTTACCCAATCCTGACACAGGGAGGTAGTGACARGAARTAACAATACGGGGCTTARTGTCTTGTAATTGGA

T. sergenti ACATCTAAGGAAGGCAGCAGGCGC-GCAAPTTACCCAATCCTGACACAGGGAGGTAGTGACARGAFATAACARTACGGGGCTTAATGTCTTGTAATTGGA

T. sergenti
Consensus

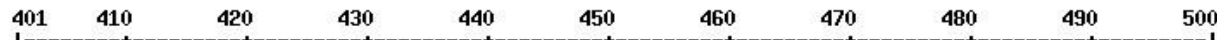

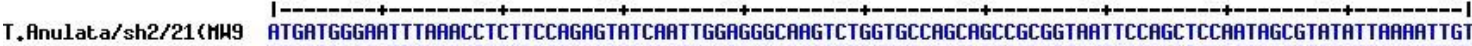

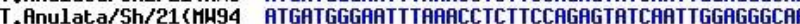

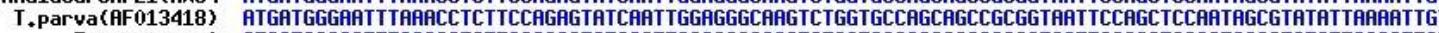
T.taurotragi ATGATGGGAATTTAAACC TCTTCCAGAGTATCAATTGGAGGGCAAGTCTGGTGCCAGCAGCCGCGGTAATTCCAGCTCCARTAGCGTATATTARAATTGT T.ovis. ATGATGGGAATTTAAACCTCTTCCAGAGTATCAATTGGAGGGCAAGTCTGGTGCCAGCAGCCGCGGTAATTCCAGC TCCAATAGCGTATATTAAAPTTG T,capreoli ATGATGGGAATTTAAACCTCTTCCAGAGTATCAATTGGAGGGCAAGTCTGGTGCCAGCAGCCGCGGTARTTCCAGCTCCAATAGCGTATATTAAFATTGT T.buffeli ATGATGGGAATTTAAACCTCTTCCAGAGTATCAATTGGAGGGCAAGTCTGGTGCCAGCAGCCGCGGTAATTCCAGCTCCAPTAGCGTATATTAAPATTGT T, sergenti ATGATGGGAATTTAAACCTCTTCCAGAGTATCAATTGGAGGGCARGTCTGGTGCCAGCAGCCGCGGTARTTCCAGCTCCAATAGCGTATATTAARATTGT

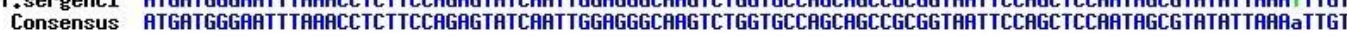

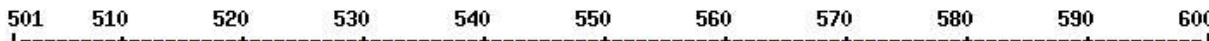

T.Anulata/sh2/21(rHM9

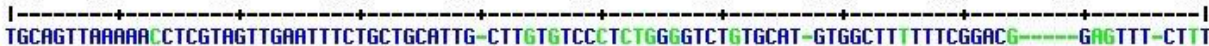

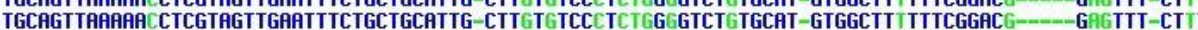
TGCAGTTAPAFAGCTCGTAGTTGAPTTTCTGCTGCATCG-CT-GTGTCCCTTCGGGTCTCTGCAT-GTGGCTTATTICRGACG----GAGTTCCCTIT TGCAGTTAAPAFGGCTCGTAGTTGAATITCTGCTGCATTG--ICGAG TCCCTCCGG GTCT TGGCAC-GTGGCTTITITCGGACG----G--ITCGCT-

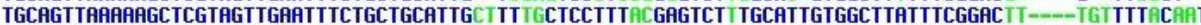

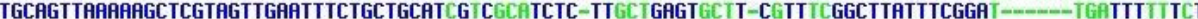

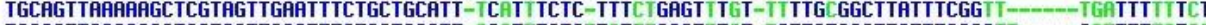

T.buffeli

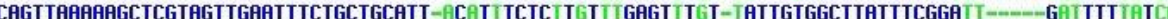

Consensus

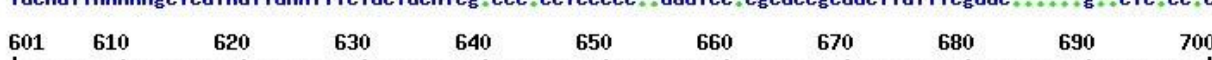

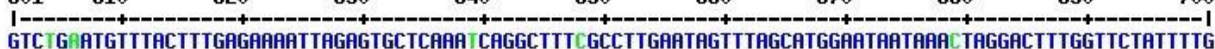

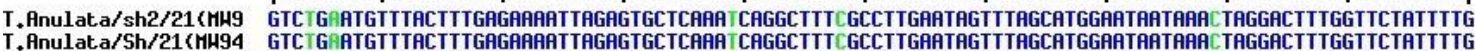

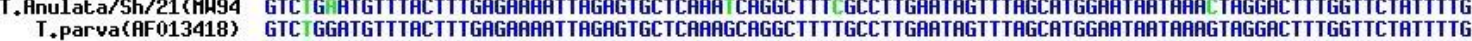

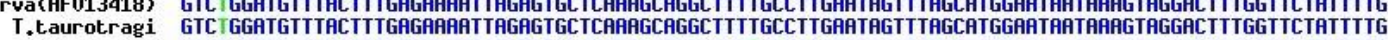

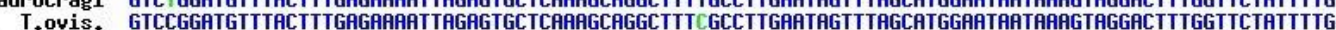

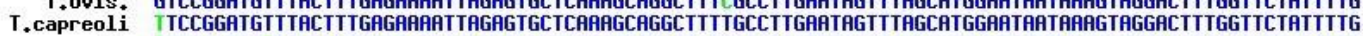

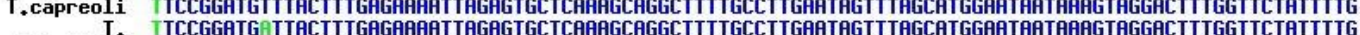

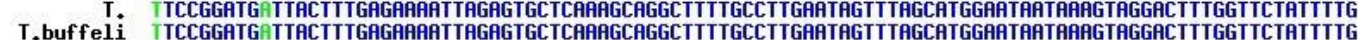

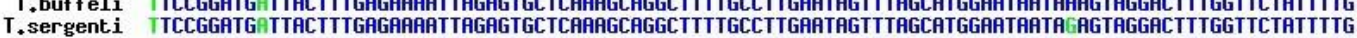

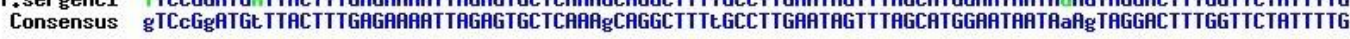

Figure 3: CLUSTAL W2.1 multiple sequence alignment of 18S rRNA gene 1098bp PCR product of two field isolate (MW940543 \& MW940544) with other Theileria spp. Strains. T.ovis (AY260172), T.taurotragi (L19082), T.capreoli (AY726011), T. sinensis (EU277003), T.buffel 


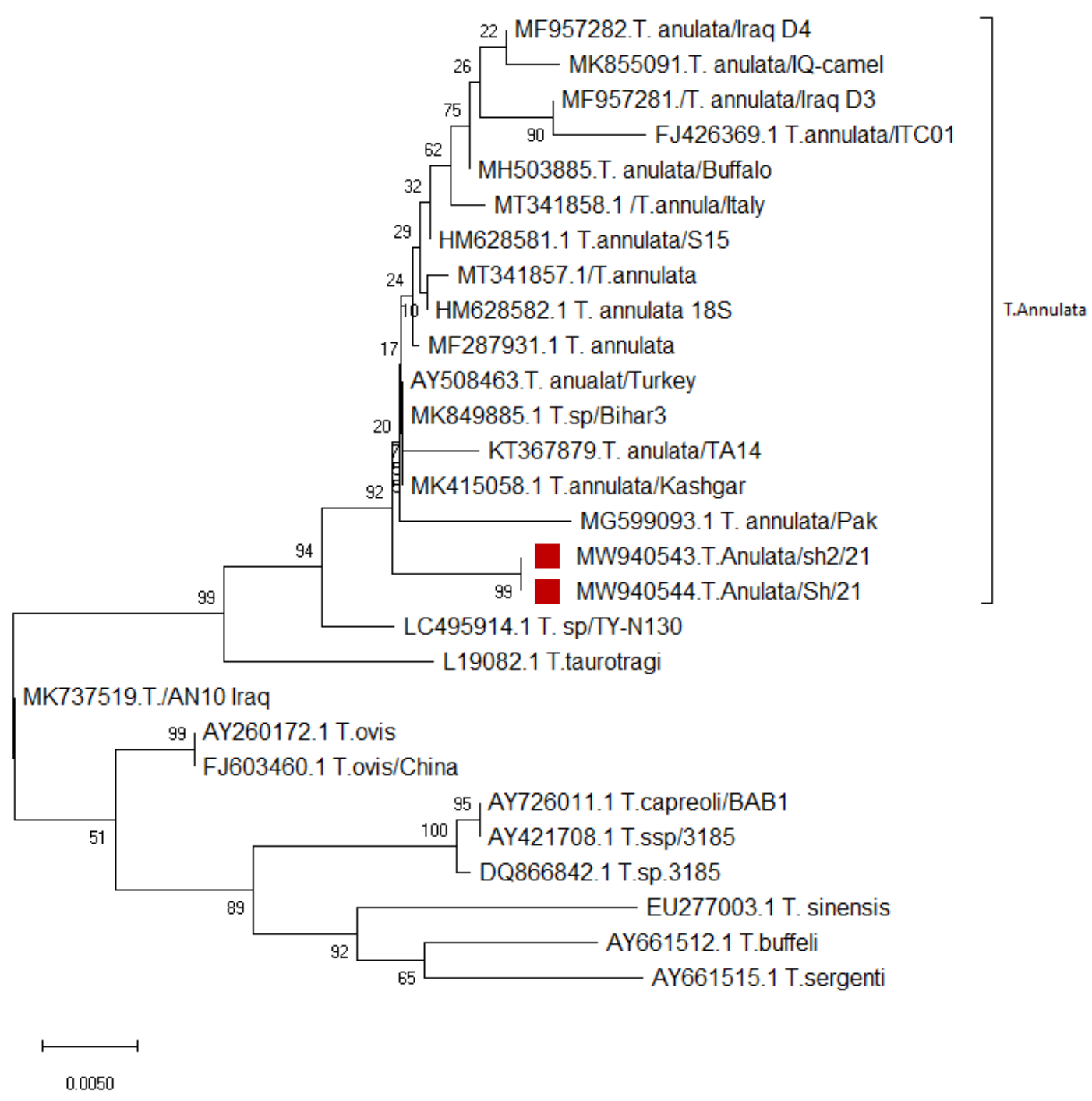

Figure 4: Neighbor-Joining method. Phylogenetic tree of Theileria spp. based on 18S rRNA gene sequences (T.anuulata,T.ovis, T. taurotragi, T. capreoli, T. sinesis, T. buffeli, and T. sergenti). The Iraqi sequences obtained in the present study are red square.

\section{Discussion}

Theileriosis is a significant clinical illness of cattle in many countries. It is also causes major economic losses as well as reduced production ${ }^{[21},{ }^{22},{ }^{23},{ }^{24]}$. Several studies in Iraq revealed that tropical theileriosis are endemic in cattle in different parts of the country ${ }^{[7},{ }^{16},{ }^{25},{ }^{26},{ }^{27]}$. The traditional identification methods are based on morphological structure inside erythrocytes. In the current study, different shapes were found inside infected erythrocytes by direct microscopic examination including ring, Oval, and parachute forms. Giemsa-stained blood smears showed that cattle erythrocytes infected with the piroplasm forms of Theileria annulata pathogens showed round-shaped appearance and irregular thorn-like protrusions (figure 1). These erythrocyte morphologic disorders are attributed to presence of the parasites in the erythrocytes ${ }^{[28]}$. They are generally in agreement with findings of ${ }^{[26]}$. However, carrier animals are sometime difficult to identify using traditional Giemsa staining method. Although, this method uses as convenient technique in the labs for the diagnosis of clinical cases, but for carrier animals' determination having low parasitemia. Thus, alternative techniques, for instance PCR can be used as it is more accurate technique to identify of persistently infected cattle with Theileria spp. ${ }^{[27},{ }^{29]}$.

According to the current study's sequence analysis, $T$. annulata was confirmed to be the source of Theileriosis in the field. The phylogenetic tree shows that two field isolates are homologous and identical to the most of other T.annulata. This demonstrates that the 18S rRNA is suitable for identification of this parasite. The disease' prevalence is lower in Kurdistan Region of Iraq (31.25\% in cattle) in comparison to other countries those used PCR technique. In Sudia Arabia, the disease' prevalence was 76.5 $\%$ in cattle ${ }^{[11]}$. In Turkey, the rate of the disease was $37.8 \%{ }^{[10]}$. Other research have shown similar prevalence rates, for instance, the rate of the disease in Iran using PCR was $31.5 \%$ in cattle ${ }^{[8]}$. On contrast to our results, according to the results of other studies, the prevalence of theileriosis was $14.32 \%$ In Dera Ismail Khan, Khyber Pakhtunkhwa Province, Pakistan ${ }^{[30]}$. In Egypt, the prevalence rate was $24.05 \%$ in cattle by PCR assay ${ }^{[12]}$, while in the Sulaibiya area of Kuwait, prevalence rate of theileriosis recorded was $7.6 \%{ }^{[31]}$, and in Spain, the prevalence rate was 22.4 $\%$ by using PCR technique ${ }^{[32]}$. These differences in the 
prevalence of $T$. annulata-infection reported from various studies can be attributed to many factors such as climatic conditions, breeds' susceptibility, vectors' distribution, breeding system, immunization, prophylactic strategy and systems of treatment. However, season is one of the most common considerable differences because in summer highest rate of the disease was recorded ${ }^{[30]}$.

\section{Conclusions}

The findings of the current study illustrate that traditional method could be a suitable technique to determine tropical theileriosis. Proper formulation of control strategies and reduction of economic loss caused by theileriosis in cattle can be performed via further identification trial of $T$. annulata based on $18 \mathrm{~S}$ rRNA gene sequences. In addition, the study showed that $T$. annulata infection was dominant among other Theileria species in cattle of the studied area. PCR technique accepted as an accurate tool to determine Theileria even in asymptomatic carrier animals which is quite necessary for carrying out of control programs successfully. Therefore, further field studies to verify these results are suggested.

\section{Conflict of interests}

None.

\section{References}

1. Kundave, V. R., Patel, A. K., Patel, P. V, Hasnani, J. J. \& Joshi, C. G. Detection of theileriosis in cattle and buffaloes by polymerase chain reaction. J. Parasit. Dis. 39, 508-513 (2015).

2. Afifi, N. A., Shihata, I. M., El-Zorba, H. Y. \& Ismail, I. M. Prevalence of theileriosis in cross-bred cattle: its detection through blood smear examination and polymerase chain reaction in Dehradun district, Uttarakhand, India. Vet. world 7, 168 (2014).

3. Demessie, Y. \& Derso, S. Tick borne hemoparasitic diseases of ruminants: A review. Adv. Biol. Res. (Rennes). 9, 210-224 (2015).

4. Mirzaei, M. Treatment of natural tropical theileriosis with the extract of the plant Peganum harmala. Korean J. Parasitol. 45, 267 (2007).

5. Maharana, B. R., Tewari, A. K., Saravanan, B. C. \& Sudhakar, N. R. Important hemoprotozoan diseases of livestock: Challenges in current diagnostics and therapeutics: An update. Vet. world $\mathbf{9}, 487$ (2016).

6. Al-Saeed, A. T. M. et al. Epidemiological studies on tropical theileriosis (Theileria annulata infection of cattle) in Kurdistan Region, Iraq. Parasitol. Res. 106, 403-407 (2010).

7. Omer, L. T., Kadir, M. A. \& Ahmed, J. S. Seroprevalence of piroplasmosis with tick distribution in northern Iraq. Iraqi J Vet Sci 26, 105-108 (2012).

8. Zadeh, S. S., Fathi, S., Dehaghi, M. M., Asl, E. N. \& Nezhad, H. A. Survey of Theileria annulata and Anaplasma marginale in cattle in Kerman area, southeast of Iran. Sci Parasitol 12, 61-66 (2011).

9. Oryan, A., Namazi, F., Sharifiyazdi, H., Razavi, M. \& Shahriari, R. Clinicopathological findings of a natural outbreak of Theileria annulata in cattle: an emerging disease in southern Iran. Parasitol. Res. 112, 123-127 (2013).

10. Dumanli, N. et al. Prevalence and distribution of tropical theileriosis in eastern Turkey. Vet. Parasitol. 127, 9-15 (2005).

11. El-Metenawy, T. M. Prevalence of blood parasites among cattle at the central area of Saudi Arabia. J. Protozool. Res. 10, 6-13 (2000).

12. Nayel, M. et al. The use of different diagnostic tools for Babesia and Theileria parasites in cattle in Menofia, Egypt. Parasitol. Res. 111, 1019-1024 (2012).
13. Friedhoff, K. \& Böse, R. Recent developments in diagnostics of some tick-borne diseases. in Expert Consultation on the Use of Applicable Biotechnological Methods for Diagnosing Haemoparasites, Merida (Mexico), 4-6 Oct 1993 (FAO, 1994).

14. Chauhan, H. C. et al. Comparison of molecular and microscopic technique for detection of Theileria annulata from the field cases of cattle. Vet. world $\mathbf{8}, 1370$ (2015).

15. Charaya, G. et al. Comparative evaluation of polymerase chain reaction assay with microscopy for detection of asymptomatic carrier state of theileriosis in a herd of crossbred cattle. Vet. world $\mathbf{9}, 1039$ (2016).

16. Alkhaled, M. J. A., A'aiz, N. N. \& Naser, H. H. Phylogenetic study of Theileria lestoquardi based on 18SrRNA gene Isolated from sheep in the middle region of Iraq. Iraqi J. Vet. Sci. 30, 27-32 (2016).

17. Kumar, S., Stecher, G. \& Tamura, K. MEGA7: molecular evolutionary genetics analysis version 7.0 for bigger datasets. Mol. Biol. Evol. 33, 1870-1874 (2016).

18. Saitou, N. \& Nei, M. The neighbor-joining method: a new method for reconstructing phylogenetic trees. Mol. Biol. Evol. 4, 406-425 (1987).

19. Felsenstein, J. Confidence limits on phylogenies: an approach using the bootstrap. Evolution (N. Y). 39, 783-791 (1985).

20. Kimura, M. A simple method for estimating evolutionary rates of base substitutions through comparative studies of nucleotide sequences. J. Mol. Evol. 16, 111-120 (1980).

21. Brown, C. G. Control of tropical theileriosis (Theileria annulata infection) of cattle. Parassitologia 32, 23-31 (1990)

22. Campbel, J. D. M. \& Spooner, R. L. Macrophages behaving badly: infected cells and subversion of immune responses to Theileria annulata. Parasitol. today 15, 10-16 (1999).

23. Omer, O. H. et al. Haematological profiles in pure bred cattle naturally infected with Theileria annulata in Saudi Arabia. Vet. Parasitol. 107, 161-168 (2002).

24. Çöl, R. \& Uslu, U. Haematological and coagulation profiles during severe tropical theileriosis in cattle. Turkish J. Vet. Anim. Sci. 30, 577-582 (2007).

25. HASSEN, Z. I. \& MEERKHAN, A. A. DETECTION AND MOLECULAR CHARACTERIZATION OF THEILERIA OVIS IN SHEEP AND GOATS WITH CLINICAL THE ILERIOSIS IN KURDISTAN, IRAQ. J. Duhok Univ. 23, 69-78 (2020).

26. Hassan, A. H. Pathological and molecular diagnostic study of theileriosis in cattle in Sulaimaniyah province, Iraq. Iraqi J. Vet. Med. 36, 306-314 (2012).

27. Al-Saeed, A. Application of Polymerase Chain Reaction (PCR) for the Detection of Theileria annulata among Cattle in Kurdistan Region/Iraq. (2009).

28. Noaman, V. Comparison of molecular and microscopic technique for detection of Theileria spp. in carrier cattle. J. Parasit. Dis. 38, $64-67$ (2014).

29. OIE. THEILERIOSIS,Technical disease cards. 1-9 https://www.oie.int/app/uploads/2021/03/theileriosis.pdf (2020).

30. Khan, A. et al. Bovine theileriosis: Prevalence, estimation of hematological profile and chemotherapy in cattle in Dera Ismail Khan, Khyber Pakhtunkhwa Province, Pakistan. Am. Sci. Res. J. Eng. Technol. Sci. 32, 8-17 (2017).

31. Ahmed, B. A., Al Hoty, K. F. \& Saddik, M. M. Theileria annulata infection in Friesian cattle imported to Kuwait from W. Germany. Vet. Med. Journal, Giza, Egypt 35, 139 146 (1987).

32. Calleja-Bueno, L. et al. Molecular, epidemiological, haematological and biochemical evaluation in asymptomatic Theileria annulata infected cattle from an endemic region in Spain. Ticks Tick. Borne. Dis. 8, 936-941 (2017). 